\title{
Corrigendum \\ Fronto-Striatal Glutamate in Autism Spectrum Disorder and Obsessive Compulsive Disorder
}

\author{
Jilly Naaijen, Marcel P Zwiers, Houshang Amiri, Steven CR Williams, Sarah Durston, Bob Oranje, \\ Daniel Brandeis, Regina Boecker-Schlier, Matthias Ruf, Isabella Wolf, Tobias Banaschewski, Jeffrey C Glennon, \\ Barbara Franke, Jan K Buitelaar, David J Lythgoe The TACTICS Consortium
}

Neuropsychopharmacology (2017) 42, 2466-2467; doi:I0.I 038/npp.2017.I63

Correction to: Neuropsychopharmacology advance online publication, 24 December 2016; doi:10.1038/npp.2016.260

Page 1: In the abstract, the sentence 'In addition, a dimensional analysis was performed, linking glutamate with a continuous measure of compulsivity across disorders' should be as follows: 'In addition, dimensional analyses were performed, linking glutamate with continuous measures related to compulsivity'. Later, in the sentence 'Dimensional analyses revealed a positive correlation between compulsive behavior (measured with the Repetitive Behavior Scale) and ACC glutamate...,' 'compulsive behavior' should read 'limited interests.'

Page 5: In 'Materials and Methods,' third paragraph under 'Statistical Analysis,' the sentence 'For metabolites that were significantly different across the groups per voxel, post hoc correlation analyses with RBS compulsivity, RBS total scores, and CBCL anxiety scores were performed...' should be as follows: 'For metabolites that were significantly different across the groups per voxel, post hoc correlation analyses with the RBS subscales and CBCL anxiety scores were performed...'

Page 5: In 'Results,' first paragraph under 'Demographics,' the sentence 'The exclusion of more participants from the striatal analyses did not influence demographic distributions in the group, regarding sex $\left(\chi^{2}=6.88, p=0.032\right)$, age $(\mathrm{F}=0.60, p=0.55)$, RBS-scores (compulsivity: $\mathrm{U}=695.5$, $p=0.012$; total: $U=621, p=0.151)$ and CBCL-anxiety $(U=430, p=0.34)$ ' should read as follows: 'The exclusion of more participants from the striatal analyses did not influence demographic distributions in the group, regarding sex $\left(\chi^{2}=6.88, \quad p=0.032\right), \quad$ age $\quad(\mathrm{F}=0.60, \quad p=0.55), \quad$ and RBS-scores (stereotypes: $U=590.0, p=0.299$; self-harm: $U=506.5, \quad p=0.932$; compulsions: $U=348.0, \quad p=0.028$; rituals: $U=528.0, p=0.834$; insisting on sameness: $U=619.5$, $p=0.154$; limited interests: $U=695.5, \quad p=0.012$; total: $U=581.5, p=0.361)$ and CBCL-anxiety $(U=430, p=0.34)$.'

Page 5: In Table 1, the rows for the RBS compulsivity score and RBS total score were incorrect and the subscales of the RBS questionnaire were omitted. The corrected RBS section of the table is provided at the end of this corrigendum.
Page 6: In 'Results,' first paragraph under 'Group Comparisons,' in the sentence 'Dimensional analysis showed a positive correlation between Glu levels in the ACC and compulsivity', 'compulsivity' should be 'limited interests'.

Pages 6-7: In 'Results,' end of the first paragraph under 'Group Comparisons,' the following passage:

'No significant correlation was observed between Glu levels in the ACC and more general repetitive behavior as measured with the RBS total (excluding the compulsivity scale) (rho $=0.08, p=0.49)$. Also no correlation was found with anxiety (rho $=-0.12, p=0.30$ ). We additionally calculated the difference between the correlations (Lee and Preacher, 2013). The correlation between compulsivity and glutamate and general repetitive behavior and glutamate differed significantly from each other $(p=0.03)$ as did the compulsivity and glutamate and anxiety and glutamate $(p=0.005)$.'

should read as follows:

'No significant correlation was observed between Glu levels in the ACC and other subscales of the RBS: stereotypes (rho $=0.18, p=0.11$ ), self-harming behavior (rho $=0.12$, $p=0.29$, compulsions (rho $=-0.01, p=0.93$ ), rituals (rho $=$ $-0.01, p=0.90$ ), and insisting on sameness (rho $=0.01$, $p=0.91)$. Also, no correlation was found with anxiety (rho $=-0.12, p=0.30$ ). The correlation between limited interests and glutamate, and between anxiety and glutamate differed significantly from each other $(p=0.005)$ (Lee and Preacher, 2013), as did the correlations between limited interests and glutamate, and between the compulsions, rituals, and insisting on sameness subscales of the RBS and glutamate (all $p$-values <0.05).'

Page 7: In the first paragraph under 'Discussion,' the sentence "We conducted both categorical analyses contrasting diagnostic groups and dimensional analyses using a continuous measure of compulsivity' should be as follows: 'We conducted both categorical analyses contrasting diagnostic groups and dimensional analyses using continuous measures of compulsivity and anxiety'.

Page 7: In the fourth paragraph under 'Discussion,' in the sentence 'This similarity across the two disorders might be due to common underlying mechanisms of compulsivity', 
Table I (excerpt) Demographic Characteristics (Based on the Group for the ACC Analysis)

\begin{tabular}{|c|c|c|c|c|c|c|c|c|}
\hline & \multicolumn{2}{|c|}{ ASD $(n=5 I)$} & \multicolumn{2}{|c|}{ OCD $(n=29)$} & \multicolumn{2}{|c|}{ Control $(n=53)$} & \multirow[t]{2}{*}{ Test statistic } & \multirow[t]{2}{*}{$p$-value } \\
\hline & Mean & SD & Mean & SD & Mean & SD & & \\
\hline \multicolumn{9}{|l|}{ RBS } \\
\hline Stereotypes & 3.26 & 3.14 & 2.83 & 2.90 & - & - & $U=792.0$ & 0.490 \\
\hline Self-harm & 1.60 & 2.34 & 1.90 & 2.97 & - & - & $U=696.5$ & 0.755 \\
\hline Insisting on sameness & 8.52 & 7.28 & 6.10 & 6.58 & - & - & $U=881.0$ & 0.111 \\
\hline Limited interests & 2.94 & 2.83 & 1.41 & 1.80 & - & - & $U=955.0$ & $0.016 * \#$ \\
\hline Total score & 24.18 & 21.27 & 21.38 & 19.65 & - & - & $U=787.5$ & 0.525 \\
\hline
\end{tabular}

'compulsivity' should read as 'compulsivity-related behaviors.' In the following sentence, 'increased compulsivity was associated' should be read as 'increased limited interests were associated'. The next sentence ('This correlation was not found with the total score on the RBS or with anxiety, which suggests that ACC glutamate is more specifically involved in compulsive behavior and not in anxiety and other aspects related to repetitive behavior, such as self-harm, resistance to change, and restricted interests') should read simply as 'This correlation was not found for the other RBS subscales or a measure of anxiety.'
Page 7: In Figure 4, the label below the $x$-axis ('Compulsivity') should be 'Limited interests.' Likewise, in the Figure legend, 'compulsivity scores' should be 'limited interests scores'.

Page 8: In the last paragraph of 'Discussion,' the sentence 'In conclusion, the current study showed increased glutamate levels in the ACC of children with compulsivity disorders compared with controls and a relation between compulsivity severity and the ACC glutamate levels', the phrase 'compulsivity severity and the ACC glutamate levels' should be as 'limited interests and the ACC glutamate levels across the disorders'. 